\title{
A Novel Immune and Inflammatory Related Gene Signature in Hepatocellular Carcinoma
}

\section{Yunji Xu}

Hengyang Medical College: University of South China https://orcid.org/0000-0001-7116-5265

\section{Guo Huang}

Hengyang Medical College: University of South China

\section{Wen bing Li ( $\triangle$ loverpig520@163.com )}

University of South China

\section{Research}

Keywords: Hepatocellular carcinoma, Immune and inflammation-related response, Overall survival, Immune status, Tumor microenvironment, Drug sensitivity

Posted Date: October 1st, 2021

DOI: https://doi.org/10.21203/rs.3.rs-927122/v1

License: (c) (i) This work is licensed under a Creative Commons Attribution 4.0 International License. Read Full License 


\section{Abstract}

Background: The prognosis of hepatocellular carcinoma (HCC) is closely related to immunity and inflammation, but the value of using immune and inflammation-related genes as predicting the prognosis of HCC requires further research.

Methods: The Hepatocellular Carcinomar mRNA data was downloaded in the TCGA and ICGC database. The R package "limma" was used to analyze the differential expression of genes (DEGs) irelated to immune and inflammatory. Univariate Cox analysis screen for immune and inflammation related genes with prognostic value, then construction and verification of the prognostic model in Hepatocellular Carcinomar. The correlation between risk score with tumor immune immersion and immune cell function was assessed through tumor microensure and immune response analysis. $\mathrm{NCl}-60$ cell line to explore the relationship between prognostic gene expression and drug sensitivity.

Results: We evaluated 8 immune and inflammatory-related genes to build a prognostic risk prediction model, riskscore is an independent risk factor affecting prognosis, closely related to histological grading and clinical staging. The immune of adCs, macrophages, Tfh cells, Treg cells and Th1 cells higher in the tumor microenvironment leads to poor prognosis of liver cancer. Using data from the $\mathrm{NCl}-60$ cell line, DNASE1L3 high expression may increased resistance of liver cancer cells to bovine platinum, solafinil and bovine platinum. The expression of SLC7A11 can increase the sensitivity of liver cancer to arsenic trioxide (ATO). Simultaneously constructing models and tumor microenvironment and drug resistance may provide effective and safe strategies for HCC chemotherapy and immunotherapy.

Conclusion:Our study screened eight immune and inflammation-related genes play an important role in HCC tumor immunity and can be used to predict the prognosis of HCC.

\section{Background}

Primary liver cancer refers to malignant tumors originating from hepatocytes and intrahepatic bile duct epithelial cells, among which hepatocellular carcinoma(HCC) is the most common. The global incidence of $\mathrm{HCC}$ is 10.1 patients per 100,000 people per year, $80 \%$ of which occur in East Asia and Africa ${ }^{[1]}$.The causes of hepatocellular carcinoma include chronic infection with hepatitis B virus and hepatitis $C$ virus,

alcoholism, non-alcoholic fatty liver, and dietary toxins such as aflatoxin ${ }^{[2]}$. In recent years, many scholars have focused on the research of liver cancer prevention and treatment strategies, including cancer risk assessment, surgical resection, liver transplantation, comprehensive treatment, etc. However, the 5-year survival rate of liver cancer patients still does not exceed $20 \%$, and the early diagnosis rate is low and high recurrence risk is still an important issue that plagues treatment ${ }^{[3-6]}$.

Inflammation is a basic pathological process. It is an immune defense mechanism produced by the body in the face of damage caused by various inflammatory factors. It is also an important part of many diseases, including malignant tumors ${ }^{[7]}$.More and more evidences have emphasized the importance of chronic inflammation in the pathobiology of liver cancer, by changing the tumor immune 
microenvironment to affect its progression, metastatic potential and treatment outcome.Studying the crosstalk between immunotherapy and inflammation has clinical significance in Central Asia for the development of new treatments and overcoming resistance ${ }^{[8]}$. Inflammation associated with cancer has been determined to be a key process in controlling tumor progression. Typical Th1-related/inflammatory mediators, such as interferon gamma, can play an important role in enhancing acquired anti-tumor immune activities in the initial stage of tumor development, and inflammation usually becomes a chronic disease that promotes tumor growth and metastasis ${ }^{[9]}$. Many malignant tumors are rich in proinflammatory cytokines and inflammatory chemokines. These different pro-inflammatory components lead to the occurrence and metastasis of primary tumors, and ultimately lead to poor survival and prognosis of patients ${ }^{[10]}$. The high infiltration of ILC2 in human melanoma is associated with a good clinical prognosis. ILC2 can coordinate the recruitment and activation of eosinophils to enhance the antitumor response. The expression of tumor-infiltrating ILC2s limits the accumulation, proliferation and antitumor effect of PD-1 in tumors. This inhibition can block PD-1 and enhance the anti-tumor response by binding to IL-33-driven ILC2 activation ${ }^{[11]}$. Epithelial Mesenchymal Transition(EMT) plays an important role in tumor progression and metastasis. A variety of inflammatory mediators, such as tumor necrosis factor, interleukin $1 \beta$, interleukin 6 , interleukin 11 and interleukin 8 , are effective inducers of EMT ${ }^{[12]}$. Cytokines, chemokines and growth factors play a leading role in the formation of the inflammatory environment in Tumor Microenvironment(TME), and promote tumor progression by directly acting on tumor cells or indirectly regulating other components of $\mathrm{TME}^{[8]}$.

Tumor immunotherapy is to control and kill tumor cells by modulating the function of the body's immune system and enhancing anti-tumor immunity. The traditional method of tumor treatment is to rely on external forces to kill tumor cells, and the target of action is tumor cells, while the current target of new tumor immunotherapy is immune cells, that is, by mobilizing the body's own immune system to inhibit or eliminate tumor cells. Tumor-associated macrophages (TAMs) are the most abundant immune cells in TME, and the increase of TAM leads to poor prognosis of HCC patients. According to different environmental stimuli, TAMs can be divided into two phenotypes: pro-inflammatory (M1) and antiinflammatory (M2). M1 TAMs act as synaptic antigen-presenting cells and play an anti-tumor effect, while M2 TAMs have higher anti-inflammatory cytokines, thereby suppressing the immune response ${ }^{[13]}$.The immune system's monitoring function plays an important role in early tumors, but immune escape and tumor microenomic disorders may be involved in tumor development ${ }^{[14]}$. Natural killer cells (NK) cells are innate immune cells that exert an anti-tumor effect by releasing cell-dissolved particles and cytokine secretion to produce direct cytotoxic effects ${ }^{[15-17]}$. NK cells play a key role in immune monitoring of normal livers, but NK cell failure and dysfunction (NK cell phethal abnormalities, differences in the identification of ligand receptors, dysfunction, and interference with other immune cells) may lead to the progression of $\mathrm{HCC}^{[18]}$.

Our research aims to study the role of Immune (humoral) and inflammatory response factors in the prognosis of liver cancer, and to find important targets related to Immune and inflammation. At the same time, we analyze the correlation between inflammatory factors and the immune system, and further verify 
through experimental and clinical studies, so as to better Improve the prognosis of hepatocellular carcinomar. The mRNA expression profile of hepatocellular Carcinomar patients and the corresponding clinical data were downloaded from public databases. Then, a multi-gene prognostic model was constructed in the TCGA cohort through the differentially expressed genes related to Immune (humoral) and inflammatory response, and this model was validated in the ICGC cohort. Finally, a functional enrichment analysis was further carried out to explore its potential biological mechanism.

\section{Results}

\section{Screen the prognostic immune and inflammation-related DEGs in the TCGA cohort}

In the TCGA cohort, 50 immune and inflammatory response-related genes were differentially expressed in tumor tissues and non-tumor tissues (Figure 1A). Univariate Cox analysis showed that 11 of them were related to OS (Figure 1B). The heatmap indicates the expression of $11 \mathrm{DEGs}$ (Figure 1C). The hazard ratio of KCNQ3 gene was $3.553(95 \% \mathrm{Cl}=1.552-8.130, \mathrm{P}=0.003$, Figure 1D). The correlation between these genes is shown in Figure $1 \mathrm{E}$.

\section{Construction and verify the stability of prognostic-related models}

Lasso-Cox regression was used to analyze the expression profiles of the above 11 genes, and a prognostic model was established according to the best value of $\lambda$, a marker containing 8 genes is determined( Figure $1 \mathrm{~F}$ and $1 \mathrm{G}$ ). The calculation method of the risk score is as follows: riskScore $=$ the express of CCNF*0.13310369848214 + the express of DNASE1L3*-0.0647265921366507 + the express of ENTPD $2 * 0.030515515064479+$ the express of MFAP2 $\quad * 0.00849895046403311+$ the express of SLC16A3*0.0466599278032965 +the express of SLC7A11*0.204041600878428 +the express of SPP1

$* 0.0496893487484051+$ the express of STMN1 $* 0.122778720315189$.According to the median value, patients were divided into high-risk groups and low-risk groups (Figure 2A). In the TCGA cohort, the highrisk group was found to be significantly related to tumor grade and stage (Table 1 ). In addition, the scatter plot shows that high-risk patients have a higher mortality rate (Figure 2B). The overall survival time of patients in the low-risk group was significantly better than that in the high-risk group(Figure $2 \mathrm{C}$ ). The prognostic model was used to generate a time-correlated ROC curve for survival prediction analysis. The area under the curve (AUC) reached 0.779 at 1 year, 0.724 at 2 years, and 0.695 at 3 years (Figure 2D).PCA analysis and t-SNE analysis showed that patients in the high- and low-risk groups were distributed in two different directions (Figure 2E-F). Verify the stability of 8 prognostic-related gene models through the ICGC cohort, and the results are consistent with the TCGA cohort, indicating that the prognostic model has good stability(Figure 2G-L).In order to explore the relationship between the expression of 8 prognostic-related genes and OS, survival analysis was performed based on the 
expression value of each gene. ENTPD2 has nothing to do with overall survival (Figure 3A-H). Except for the high expression of DNASE1L3, which indicates that overall survival is higher than the low expression group, the remaining 7 genes are in tumor tissues low expression is beneficial to the overall survival of patients (Figure 3I-P).

\section{TABLE 1}

Baseline characteristics of patients in the high- and low-risk risk group.

\begin{tabular}{|c|c|c|c|c|c|c|}
\hline \multirow[t]{2}{*}{ Characteristics } & \multicolumn{3}{|l|}{ TCGA-LIHC } & \multicolumn{3}{|l|}{ ICGC-LIRP } \\
\hline & High Risk & Low Risk & Pvalue & High Risk & Low Risk & Pvalue \\
\hline \multicolumn{7}{|l|}{ Age } \\
\hline$\leq 65$ & $112(61.54 \%)$ & $115(62.84 \%)$ & 0.8817 & $44(38.26 \%)$ & $45(38.79 \%)$ & 1 \\
\hline$>65$ & $70(38.46 \%)$ & $68(37.16 \%)$ & & $71(61.74 \%)$ & $71(61.21 \%)$ & \\
\hline \multicolumn{7}{|l|}{ Gender } \\
\hline FEMALE & $60(32.97 \%)$ & $59(32.24 \%)$ & 0.971 & $29(25.22 \%)$ & $32(27.59 \%)$ & 0.7956 \\
\hline MALE & $122(67.03 \%)$ & $124(67.76 \%)$ & & $86(74.78 \%)$ & $84(72.41 \%)$ & \\
\hline \multicolumn{7}{|l|}{ Grade } \\
\hline G1-2 & $98(53.85 \%)$ & 132(72.13\%) & $\begin{array}{l}2.00 \mathrm{E}- \\
04\end{array}$ & & & \\
\hline G3-4 & $83(45.6 \%)$ & $47(25.68 \%)$ & & & & \\
\hline unknow & $1(0.55 \%)$ & $4(2.19 \%)$ & & & & \\
\hline \multicolumn{7}{|l|}{ Stage } \\
\hline Stage I-II & $112(61.54 \%)$ & $142(77.6 \%)$ & $\begin{array}{l}9.00 \mathrm{E}- \\
04\end{array}$ & $56(48.7 \%)$ & $85(73.28 \%)$ & $\begin{array}{l}2.00 \mathrm{E}- \\
04\end{array}$ \\
\hline Stage III-IV & $57(31.32 \%)$ & $30(16.39 \%)$ & & $59(51.3 \%)$ & $31(26.72 \%)$ & \\
\hline unknow & $13(7.14 \%)$ & $11(6.01 \%)$ & & & & \\
\hline
\end{tabular}

\section{Independent prognostic analysis of 8 genes}

Univariate and multivariate Cox analyses was performed on clinical indicators to determine whether the risk score can be used as an independent prognostic predictor of overall survival.Univariate Cox analysis show the riskScores in TCGA and ICGC cohorts were signifificantly correlated with OS (TCGA cohort: HR = 4.754, 95\% Cl = 2.888-7.824, $\mathrm{P}<0.001$; ICGC cohort: $\mathrm{HR}=4.108,95 \% \mathrm{Cl}=2.221-7.633, \mathrm{P}<0.001$ ) (Figures $4 A, D)$. Multivariate Cox analysis shows that risk score is still an independent predictor of OS (TCGA 
cohort: $\mathrm{HR}=4.210,95 \% \mathrm{Cl}=2.430-7.293, \mathrm{P}<0.001$; ICGC cohort: $\mathrm{HR}=3.189,95 \% \mathrm{Cl}=2.211-6070, \mathrm{P}<$ 0.001 ) (Figures $4 B, E) . R O C$ curve analysis showed that in the TCGA data set (AUC=0.782) and the ICGC data set $(\mathrm{AUC}=0.730)$ (Figure $4 \mathrm{C}, \mathrm{F})$, the risk score has good predictive accuracy for the prognosis in Hepatocellular Carcinomar.

\section{The relationship between the riskScore of the prognostic model and the clinical characteristics}

By analyzing the correlation between the riskscore and the clinical characteristics of HCC patients(Table 1 ), we found that the histological grade 3-4 ( $<<0.001)$, the TNM stage III-IV $(P<0.001)$, the risk score was significantly higher than the histological grade 1-2 or TNM stages I-II (Figure 5C, D). In addition, the risk score of TNM stages III-IV in the ICGC data set is significantly higher than that of TNM stage I-II (there is no data on HCC classification in the ICGC data set) (Figure 5G). Extract the results of multi-factor analysis from the TCGA cohort and ICGC cohort data to establish a nomogram, verify the scores of 8 independent prognostic factors, find the corresponding probabilities on the nomogram, and estimate the patient's 1 year Probability of survival at 2 and 3 years. The nomogram performs well in predicting 1,2, and 3-year survival probabilities(Figure 6).

\section{Tumor microenvironment and immune status analysis}

In order to further explore whether there are differences in the immune-related status between the high-risk and low-risk groups, different immune cells and related immune pathways were scored by SSGSEA analysis. In terms of the antigen presentation process, it was found that aDCs, Macrophages, HLA, MHCclass-I, Parainflammation were significantly different between the high-risk and low-risk groups, and the immune score of the high-risk group was higher than that of the low-risk group(Figure7A,B). Compared with the low-risk group, the immune scores of Tfh cells, Treg cells and Th1 cells in the high-risk group were higher, indicating that there are differences in $T$ cell regulation between the high-risk group and the low-risk group. In addition, the high-risk group had higher CCR and checkpoint scores, while the type II IFN reactivity was just the opposite. In the low-risk group, the immune scores of B cells, mast cells, neutrophils and NK cells were higher, indicating that there are differences in immune regulation between the high-risk group and the low-risk group.In the high and low risk groups, the results of the ICGC cohort are similar to the results of the TCGA cohort(Figure 7C,D)

In order to understand the correlation between immune components and risk scores, we conducted a correlation analysis between immune infiltration and risk scores. There are six types of immune infiltration in human tumors, corresponding to tumors promoted to tumor suppression: $\mathrm{C} 1$ (wound healing), C2 (INF-y dominant), C3 (inflammation), C4 (lymphocytes) Depletion), C5 (immune quietness) and $\mathrm{C} 6$ (TGF- $\beta$ dominant). No patient sample in HCC belongs to the $\mathrm{C} 5$ immune subtype, and there is only 1 sample from $\mathrm{C} 6$. Therefore, the $\mathrm{C} 5$ and $\mathrm{C} 6$ immune subtypes are not included in this study. In order to 
further analyze the correlation between the HCC risk score and immune infiltration in the TCGA data, it was found that the high risk score was significantly correlated with $\mathrm{C} 1$, while the low risk score was significantly correlated with C3 (Figure 7E). Tumor stemness can be measured by DNA stemness of DNA methylation pattern (DNAss) and RNA stemness score (RNAss) score of gene expression. The matrix score and immune score are used to evaluate the tumor immune microenvironment. In order to explore whether there is a correlation between the risk score and tumor stem cells and the immune microenvironment, the results showed that the risk score RNAss was not significantly correlated, and it was positively correlated with DNAss $(P<0.001)$, immune score $(P=0.024)$ and negatively correlated with matrix score $(P=0.012)(($ Figure $7 F-1)$.

Prognostic gene expression also has a certain correlation with stromal score and immune score. CCNF, DNASE1L3, ENTPD2, MFAP2, SLC16A3, SPP1, STMN1 have a strong correlation with Stromal score. DNASE1L3, MFAP2, SLC16A3, and SPP1 are closely related to the immune score(Figure 8).

PD1/PD-L1 and CTLA4 are classic immune checkpoints, which play an inhibitory role under physiological conditions to regulate the duration and intensity of immune response, and participate in assisting tumor immune escape in the tumor microenvironment. The expression levels of PD-L1 and CTLA4 in the highrisk group were significantly higher than those in the low-risk group (Figure 9A, B), and the expression levels of these immune checkpoints were positively correlated with the risk score.The expression of other immune checkpoints, such as TLT8, CXCR2, GPC3, also increased in the high-risk group, and the expression level was positively correlated with the risk score. Among them, the GPC3-derived peptide vaccine showed good anti-tumor efficacy.

\section{Functional enrichment analysis}

The GO function and KEGG pathway enrichment analysis between the high-risk and low-risk groups were completed through R. GO function enrichment analysis showed that nuclear division, chromosome segregation, steroid hydroxylase activity. KEGG enrichment analysis main pathways involved were Cell cycle, Human T-cell leukemia virus 1 infection, HIF-1 signaling pathway, PPAR signaling pathway, IL-17 signaling pathway, PI3K-Akt signaling pathway, Central carbon metabolism in cancer(Figure 10A, B). The results of the ICGC cohort are similar to the results of the TCGA cohort(Figure 10C, D)

\section{Prognostic gene expression and sensitivity of cancer cells to chemotherapy}

The expression of eight prognostic-related genes in the $\mathrm{NCl}-60$ cell line was studied, and the relationship between the expression levels of eight prognostic genes and drug sensitivity was analyzed. We selected the first 16 significant differences in the $p$-value to analyze the correlation between gene and drug

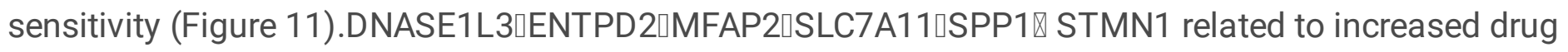

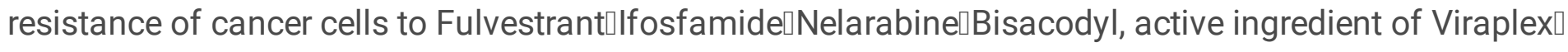




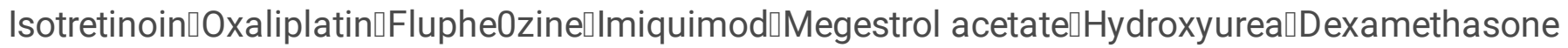
Decadron₫Epirubicin₫Carmustine.

Interestingly, the increase in SLC16A3 expression is associated with the increase in the sensitivity of cancer cells to Arsenic trioxide and Ixazomib citrate.

\section{Discussion}

The incidence of primary liver cancer ranks 6 th among all tumors and 4th among cancer-related deaths. In 2018, there were approximately 841,000 new cases of primary liver cancer worldwide, and 782,000 deaths related to liver cancer ${ }^{[23]}$. Hepatocellular carcinoma (HCC) accounts for about $75-85 \%$ of primary liver cancer. Most HCC patients have reached the advanced stage when they are diagnosed, lose surgical opportunities and have a poor prognosis. The 5 -year overall survival rate (OSR) is $20 \%{ }^{[24]}$. The main treatments for $\mathrm{HCC}$ include surgical resection, liver transplantation, transarterial chemoembolization (TACE) and radiofrequency ablation (RFA), supplemented by systemic chemotherapy and targeted drug therapy ${ }^{[25]}$. Because early liver cancer no obvious symptoms or atypical clinical manifestations, more than $80 \%$ of patients are already in the advanced stage at the time of diagnosis and cannot receive radical treatment ${ }^{[26]}$.Alpha-fetoprotein (AFP) is the most commonly used biomarker in HCC monitoring and diagnosis, but it has limited specificity and sensitivity in detecting early HCC [27]. The combination of AFP and serum GP73 is better than AFP alone in the early screening of HCC ${ }^{[28]}$.Serum AFP and AFP-L3 combined with ultrasonography can significantly improve the sensitivity of liver cancer diagnosis (94.3\%) [29].Using serum AKR1B10 alone to diagnose early HCC has a sensitivity of $61 \%$ and a specificity of $86 \%$, which is better than AFP alone. The combination of the two AUROC is as high as $94 \%$, which can be vigorously promoted as a marker for early liver cancer diagnosis ${ }^{[30]}$. However, there are no relevant reports on the immune and inflammation-related markers in serum. At present, the prediction of liver cancer models related to iron death, DNA methylation, m6A methylation, long non-coding RNA, endoplasmic reticulum stress and glycolysis has good predictive effects ${ }^{[31-36]}$. The prognostic model constructed by immune and inflammation-related genes screened in this study has an AUC of $77.9 \%$ (Figure2D), which also has a good predictive effect.

In this study, we analyzed the expression of 526 immune and inflammatory response-related genes in HCC tissues, and selected 50 DEGs from the TCGA cohort (Figure 1A). In univariate Cox analysis, 11 DEGs were associated with OS (Figure 1C, D).Prognostic models of 8 immune and inflammatory responserelated genes were constructed through LASSO regression analysis and verified in the ICGC cohort. According to the median risk score, patients were divided into high-risk groups and low-risk groups. Independent prognostic analysis showed that risk score is an independent predictor of OS (Figure4).

The prognosis model established in this study includes 8 immune and inflammatory response related genes (CCNF, DNASE1L3, ENTPD2, MFAP2, SLC16A3, SLC7A11, SPP1, STMN1). Except for DNASE3L3, these genes are all up-regulated in HCC tumor tissues and are associated with poor prognosis (Figure3).Cyclin F (CCNF) has been shown to regulate the cell dNTP pool and maintain the stability of the 
genome by interacting with ribonucleotide reductase family member 2 (RRM2) ${ }^{[37]}$. Patients with low CCNF expression may have a shorter survival time than those with high expression ( $P=0.001)$, and have a higher tendency to relapse $(P=0.037){ }^{[38]}$. DNASE1L3 is very important for DNA catabolism and apoptosis. It combines with DNASE1 and plays a key role in the neutrophil extracellular trap and cfDNA degradation, thereby reducing organ damage after inflammation ${ }^{[39]}$. The up-regulated expression of DNASE1L3 alleviates the accumulation of cytoplasmic DNA under DDR activation conditions, which in turn leads to cell senescence and SASP inhibition, and tumor angiogenesis is impaired. These results indicate that DNASE1L3 is a potential biomarker for predicting the prognosis of $\mathrm{HCC}^{[40]}$, and the positive expression of DNASE1L3 can be used as a key indicator of a good prognosis after liver cancer ${ }^{[41]}$. E-NTPDases are transmembrane exonucleases on the surface of CD39 superfamily cells, which regulate inflammation and tissue repair by catalyzing the phosphohydrolysis of extracellular nucleotides and regulating purine signaling ${ }^{[42]}$. IL-6 transcription down-regulates the expression of ENTPD2 in portal vein fibroblasts without inducing myofibroblast differentiation ${ }^{[43]}$. Microfibril-associated protein 2 (MFAP2), also known as microfibril-associated glycoprotein 1 (MAGP1), is a component of extracellular elastic microfibrils and interacts with fibrin to affect the function of microfibrils ${ }^{[44]}$. MFAP2 is significantly up-regulated in HCC, and may promote the development and progression of HCC through its interaction with mutant fibrillin-1. DNMTs inhibitors can down-regulate MFAP2, and MFAP2 may become a potential immunotherapy target for HCC patients ${ }^{[45]}$.SLC16A3 has been proven to be a downstream factor of TSTA3 immune responsemediated metabolic coupling cell cycle and non-neoplastic hepatitis/cirrhosis tissue replication ${ }^{[46]}$. The SLC7A11 gene is located on human chromosome 4 and contains 14 exons. It is widely expressed in tissues and cells such as brain, liver, macrophages and retinal pigment cells. Down-regulation of SLC7A11 in HCC cells may increase intracellular ROS levels. In order to inhibit cell proliferation in vitro, the overexpression of SLC7A11 may be related to the advanced pathological stage of liver cancer ${ }^{[47]}$. Secreted phosphoprotein 1 (SPP1) In tumors, alternative splicing variants are associated with many malignant characteristics in cancer, such as epithelial-mesenchymal plasticity, cancer cell stem cell resistance, chemotherapy resistance and radioresistance, in HCC Among them, SPP1 is an effective prognostic biomarker, which may induce chemotherapy resistance by regulating the autophagy of HCC cells ${ }^{[48]}$. In HCC, the high expression of STMN1 is positively correlated with higher AFP levels, tumor size, vascular invasion and intrahepatic metastasis, lower 5-year survival rate and early recurrence rate. The MET inhibitor crizotinib can effectively inhibit the crosstalk between cancer cells and stellate cells caused by the HGF/MET pathway triggered by STMN1, and can effectively slow down the tumor growth caused by the high expression of stmn $1^{[49]}$.

In order to understand the relationship between risk scores and immune cells and functions, we studied the role of risk scores in the types of immune infiltration. The results showed that the high-risk score was significantly related to $\mathrm{C} 1$, while the low-risk score was clearly related to C3 (inflammation) (Figure?), suggesting that $\mathrm{C} 1$ promotes the occurrence and development of tumors, and $\mathrm{C} 3$ is a good protective factor. It shows that immunity and inflammation play an important role in tumors. In terms of the association between risk score and clinical characteristics, we found that the overall OS of the high-risk 
group, Grade3-4 and StagellI-IV was significantly shortened (Figure7E).In KEGG enrichment analysis, the main signaling pathways, cell cycle, human T-cell leukemia virus 1 infection, HIF-1 signaling pathway, PPAR signaling pathway, IL-17 signaling pathway, PI3K-Akt signaling pathway, cancer metabolism are closely related to tumor occurrence and development the pathways are significantly enriched in high risk (Figure10).

In the high-risk group, the immune scores of aDCs, macrophages, Tfh cells, Treg cells and Th1 cells were higher (Figure7). There are a large number of inflammatory cells infiltrated in tumor tissues, among which macrophages have tumor-promoting and immunosuppressive effects ${ }^{[50]}$. The immunosuppression of Tfh cells, Treg cells and Th1 cells in the tumor microenvironment leads to poor prognosis of liver cancer ${ }^{[51]}$.Programmed cell death protein-1 (PD-1) is mainly expressed on the surface of activated T cells, B cells and macrophages. PD-1 inhibits the activation of antigen-specific T cells by binding to its ligands PD-L1 and PD-L2. When PD-L1, which is highly expressed on the surface of tumor cells, binds to PD-1 on the surface of immune cells, it will cause tumor immune escape ${ }^{[52]}$.CTLA-4 inhibitors induce T cell anergy, thereby inhibiting the anti-tumor immune response ${ }^{[53]}$. TLR7/8 agonists promote NK-DC crosstalk and enhance the anti-tumor effect of NK cells in hepatocellular carcinoma ${ }^{[54]}$. SGPC3 attenuates the antitumor activity of CAR-T cells in vitro and in vivo, possibly through the combination of membrane-bound GPC3 and CAR-T cells, which leads to immune escape ${ }^{[55] .}$

Cancer stem cells (CSCs) have been shown to be the cause of HCC ${ }^{[56]}$ recurrence, metastasis, and resistance to local and systemic treatments. Among them, the HIF-1 signaling pathway, Akt signaling pathway and IL-17 signaling pathway lead to an increase in the proportion of CSCs in liver cancer tissues, leading to immunosuppression ${ }^{[57]}$. Our research found that MFAP2 is negatively correlated with RNAss and DNAss, and STMN1 is positively correlated with RNAss and DNAss. It shows that MAPF2 may inhibit the differentiation of cancer stem cells, and STMN1 promotes the differentiation of cancer stem cells, but its mechanism of promoting tumor proliferation and invasion is worthy of further study. (Figure8), but they promote tumor proliferation and invasion. The specific mechanism is worthy of further study. Prognostic gene expression also has a certain correlation with stromal score and immune score. CCNF, DNASE1L3, ENTPD2, MFAP2, SLC16A3, SPP1, STMN1 have a strong correlation with Stromal score, indicating that they may be secreted by stromal cells or involved in stromal correlation. DNASE1L3, MFAP2, SLC16A3, and SPP1 are closely related to the immune score, indicating that they are closely related to tumor immunity. This study found that prognostic gene expression also has a certain correlation with Stromal score, immune score and ESTIMATE score. DNASE1L3, MFAP2, SLC16A3 and SPP1 are positively correlated with interstitial score and immune score, indicating that they may be related to interstitial cells and immune cells ${ }^{[58-60]}$.

Using data from the $\mathrm{NCl}-60$ cell line, we found that the increased expression of some prognostic genes is associated with the increased resistance of some FDA-approved chemotherapeutic drugs, such as oxaliplatin, cyclinamide, epirubicin, nelarabine and fluoride. Of course, various prognostic genes are also related to the increase in drug sensitivity of some drugs. 
For example, the increased expression of DNASE1L3 and the resistance of liver cancer cells to oxaliplatin, sorafenib combined with oxaliplatin, fluorouracil and calcium folinate in the treatment of portal vein invasion of hepatocellular carcinoma and clinical trials ${ }^{[61]}$. The expression of SLC7A11 can increase the sensitivity of liver cancer to arsenic trioxide (ATO). Studies have reported that ATO can effectively induce the differentiation of CSCs by down-regulating CSC-related genes, inhibiting the tumorigenic ability of CSCs. The combination therapy of ATO and 5-FU/cisplatin can significantly improve the therapeutic effect of liver cancer cells. ATO and 5-FU/cisplatin synergistically inhibit LIF/JAK1/STAT3 and NF-kB signaling pathways are the potential molecular mechanisms of their differentiation ${ }^{[62]}$.

\section{Conclusion}

Our study screened eight immune and inflammatory response-related genes and constructed a new and stable risk model. The TCGA and ICGC cohorts verified that the model is closely related to the OS of HCC patients and can detect the risk level of HCC. The eight genes of the risk model may play a role in the development and progression of HCC, and the underlying mechanisms of immunity and inflammation are worthy of further study. Simultaneously constructing models and tumor microenvironment and drug resistance may provide effective and safe strategies for HCC chemotherapy and immunotherapy.

\section{Materials And Methods}

\section{Hepatocellular Carcinomar related data and Immune and inflammation-related Genes}

The Hepatocellular Carcinomar mRNA data for 370 patients and the corresponding clinical data was downloaded in The TCGA (https://portal.gdccancer.gov/repository) database in a standardized FPKM format as the training group data. Then downloaded the data from the International Cancer Genome Consortium (ICGC) Database (https://dcc.icgc.org/) of the International Union for Hepatocellular Carcinomar,including mRNA-seq data from 231 patients and clinical information.All data of TCGA and ICGC were public. We follow the data access policies and release guidelines of TCGA and ICGC. Our study obtained 546 immune(humoral) and inflammation related genes(Systematic name: M8838) were found in the molecular signature database(http://www.gsea-msigdb.org/gsea/).

\section{Construction and verification of the prognostic model in Hepatocellular Carcinomar}

In the TCGA cohort, the R package "limma" was used to analyze the differential expression of genes (DEGs) in tumor tissues and paracancerous tissues to screen out differential genes related to immune and inflammation. The screening threshold is $\left|\log _{2} F C\right|>2$, and FDR $<0$. 05. At the same time, a Univariate Cox analysis of overall survival (OS) was performed to screen for immune and inflammation related genes with prognostic value. Use the R package "venn" to intersect the iron death-related genes with differences and prognostic value. In order to achieve the purpose of minimizing the risk of overfitting, the 
author uses Lasso Cox regression analysis and combines the R package "glmnet" to construct a prognostic model ${ }^{[19,20]}$. The independent variable in the regression analysis is the gene expression at the intersection of difference and prognosis, and the dependent variable is the overall survival rate and status of breast cancer patients in the TCGA cohort, and the gene of the optimal model and the corresponding regression coefficient are obtained.Calculate the risk score of each patient according to the standardized expression level of each gene and its corresponding regression coefficient [risk score $=\mathrm{e} \mathbb{\mathbb { }}$ (gene 1 expression $\times$ corresponding regression coefficient $+\ldots+$ gene $n$ expression $\times$ corresponding regression coefficient] According to the obtained risk score to find the median, the patients are divided into high-risk group and low-risk group. In the survival analysis, the R package "survminer" is used to construct the survival curve according to the high-risk and low-risk groups. The R package "timeROC" is used to perform ROC curve analysis to evaluate the predictive ability of its characteristic gene prognostic model. Principal component analysis (PCA) and t-distributed random neighbor embedding (t-SNE) analysis are used, and the two-dimensional visualization data is realized with the "Rtsne" and "ggplot2" packages. Univariate and multivariate Cox regression analysis were used, and the "survival" package was used to determine independent prognostic factors. The nominal graph is used to predict the survival probability of HCC patients with R package "rms".

\section{Functional enrichment analysis}

Use gene set enrichment analysis (GSEA) to perform GO and KEGG function enrichment analysis on DEGs between high-risk and low-risk groups, and perform function enrichment analysis on Hallmark genes. Single-sample gene set enrichment analysis (ssGSEA) calculated 16 immune infiltration scores and the activity of 13 immune-related pathways between high and low risk groups based on the $\mathrm{R}$ package "gsva" [21].

\section{Tumor microenvironment and immune response analysis}

Immune score, matrix score and comprehensive score are generated using the ESTIMATE package loaded in $\mathrm{R}$ language (version 4.0.3) to estimate the ratio of immunity, matrix components and the sum of the two in TME for each sample, with 3 scores formal performance: Immune Score, StromalScore and Estimate Score, which respectively represent the scores of the immune component, matrix component and the sum of the two components, that is, the higher the respective score, the greater the proportion of the corresponding component in the TME ${ }^{[22]}$.

\section{Drug sensitivity analysis}

$\mathrm{NCl}-60$ data of 60 different cancer cell lines from 9 different tumors were collected through CellMiner (https://discover.nci.nih.gov/cellminer).Pearson correlation analysis was performed to explore the relationship between prognostic gene expression and drug sensitivity. Related analysis of the efficacy of 263 drugs or clinical trials approved by the FDA.

\section{The establishment of forecast nomogram}


The 8 predictive genes determined by multiple regression analysis were used as independent prognostic factors to construct a nomogram. Use survival and time-related genes to construct a specific model and connect to the ROC curve to determine the accuracy of the prediction model for 1 year, 2 years, and 3 years. Use the calibration chart and the consistency index ( $\mathrm{C}$ index) to correct the nomogram (through the guidance method of 1000 resampling).

\section{Statistical Analysis}

WilCoxon test was used to compare DEGs between tumor tissue and normal tissue. The test of different proportions adopts the chi-square test. The Mann-Whitney test was used to compare the SSGSEA scores of immune cells or immune pathways between the high and low risk groups, and the $\mathrm{BH}$ method was used to adjust the $\mathrm{P}$ value. The Kaplan-Meier analysis method was used to compare the differences in OS between different groups. Univariate and multivariate Cox analysis were used to screen independent predictors of OS. Spearman or Pearson correlation analysis was used to detect the correlation of prognostic model risk score or prognostic gene expression level with dryness score, interstitial score, immune score and drug sensitivity. Using R software (version 4.0.3), packages venn, igraph, ggplot2, pheatmap, ggpubr, corrplot and survminer are used to create modules. In all statistical results, a two-tailed $P$ value less than 0.05 indicates statistical significance.

\section{Abbreviations}




\begin{tabular}{|ll|}
\hline HCC & Hepatocellular Carcinoma \\
\hline OS & Overall Survival \\
\hline EMT & Epithelial Mesenchymal Transition \\
\hline TME & Tumor Microenvironment \\
\hline ATO & Arsenic Trioxide \\
\hline TAMs & Tumor-associated macrophages \\
\hline ICGC & International Cancer Genome Consortium \\
\hline TCGA & The Cancer Genome Atlas \\
\hline DEGs & Differential expression of genes \\
\hline PCA & Principal component analysis \\
\hline t-SNE & t-distributed random neighbor embedding \\
\hline GSEA & gene set enrichment analysis \\
\hline SSGSEA & Single-sample gene set enrichment analysis \\
\hline C-index & Consistency index \\
\hline TACE & Transarterial chemoembolization \\
\hline RFA & Radiofrequency ablation \\
\hline AFP & Alpha-fetoprotein \\
\hline CCNF & Cyclin F \\
\hline DNASE1L3 & Deoxyribonuclease I-like 3 \\
\hline ENTPD2 & Ectonucleoside triphosphate diphosphohydrolase 2 \\
\hline MFAP2 & Microfibril-associated protein 2 \\
\hline SLC16A3 & Solute carrier family 16 (monocarboxylate transporter), member 3 3 Programmed cell death protein-1 \\
\hline SPP1 & Secreted phosphoprotein 1 \\
\hline STMN1 & Stathmin 1 \\
\hline RRM2 & Ribonucleotide reductase family member 2 \\
\hline PDCs & Cancer stem cells \\
\hline
\end{tabular}

\section{Declarations}




\section{Acknowledgments}

We would like to acknowledge the TCGA and IGGC network for providing data.

\section{Authors Contributions}

WL conceived and designed the project. $\mathrm{YX}$ and $\mathrm{GH}$ acquired the data to analyzed and interpreted the data. YX wrote the paper. $W L$ revised the paper.

\section{Funding}

No funding for our research.

\section{Data available}

All data in this study can be obtained by contacting the corresponding author.

The raw data of this study are derived from the TCGA database (https://portal.gdc.cancer.gov/) andlnternational Cancer Genome Consortium (ICGC) Database (https://dcc.icgc.org/), which are publicly available databases.

\section{Ethics approval and consent to participate}

Not necessary.

\section{Consent for publication}

Not applicable.

\section{Competing Interests}

All authors have declared that no competing interest exists.

\section{References}

1. Siegel RL, Miller KD, Jemal A. Cancer Statistics, 2017[J]. CA Cancer J Clin. 2017;67:7-30.

2. Yang JD, Hainaut P, Gores GJ, Amadou A, Plymoth A, Roberts LR. A global view of hepatocellular carcinoma: trends, risk, prevention and management. Nat Rev Gastroenterol Hepatol. 2019;16(10):589-604. 
3. El-SERAG, H B. RUDOLPH K L. Hepatocellular carcinoma:epidemiology and molecular carcinogenesis[J]. Gastroenterology,2007, 132(7): 2557-2576.

4. YANG JD, HAINAUT P, GORES $G \mathrm{~J}$, et al. A global view ofhepatocellular carcinoma: trends, risk, prevention and management[J]. Nat Rev Gastroenterol Hepatol. 2019;16(10):589-604.

5. ZHANG H, YE Y, LI W. Perspectives of molecular therapy-targeted mitochondrial fission in hepatocellular carcinoma[J/OL]. Biomed Res Int, 2020: 1039312.

6. $\mathrm{CHOI} \mathrm{C,} \mathrm{YOO} \mathrm{G} \mathrm{S,} \mathrm{CHO} \mathrm{W} \mathrm{K,} \mathrm{et} \mathrm{al.} \mathrm{Optimizing} \mathrm{radiotherapy} \mathrm{with} \mathrm{immune} \mathrm{checkpoint} \mathrm{blockade} \mathrm{in}$ hepatocellular carcinoma[J]. World J Gastroenterol. 2019;25(20):2416-29.

7. Karin $\mathrm{M}$, Clevers $\mathrm{H}$. Reparative inflammation takes charge of tissue regeneration[J]. Nature. 2016;529(7586):307-15.

8. Hibino S, Kawazoe T, Kasahara H, et al. Inflammation-Induced Tumorigenesis and Metastasis[J]. Int J Mol Sci, 2021,22(11).

9. Zaidi MR. The Interferon-Gamma Paradox in Cancer[J]. J Interferon Cytokine Res. 2019;39(1):30-8.

10. Crusz SM, Balkwill FR. Inflammation and cancer: advances and new agents[J]. Nat Rev Clin Oncol. 2015;12(10):584-96.

11. Jacquelot N, Seillet C, Wang M, et al. Blockade of the co-inhibitory molecule PD-1 unleashes ILC2dependent antitumor immunity in melanoma[J]. Nat Immunol, 2021.

12. Sistigu A, Di Modugno F, Manic G, et al. Deciphering the loop of epithelial-mesenchymal transition, inflammatory cytokines and cancer immunoediting[J]. Cytokine Growth Factor Rev. 2017;36:67-77.

13. Xiao N, Zhu X, Li K, et al. Blocking siglec-10(hi) tumor-associated macrophages improves anti-tumor immunity and enhances immunotherapy for hepatocellular carcinoma[J]. Exp Hematol Oncol. 2021;10(1):36.

14. Liu Y, Cao X. Immunosuppressive cells in tumor immune escape and metastasis[J]. J Mol Med. 2016;94:509-22.

15. Mandal A, Viswanathan C. Natural killer cells: in health and disease[J]. Hematology/Oncology and Stem Cell Therapy,2015,8: 47-55.

16. Abel AM, Yang C, Thakar MS, Malarkannan S. Natural killer cells: development, maturation, and clinical utilization[J]. Front Immunol. 2018;9:1869.

17. Morvan MG, Lanier LL. NK cells and cancer: you can teach innate cells new tricks[J]. Nat Rev Cancer. 2016;16:7-19.

18. Han C, Jiang $Y$, Wang $Z$, et al. Natural killer cells involved in tumour immune escape of hepatocellular carcinomar[J]. Int Immunopharmacol. 2019;73:10-6.

19. Liu C, Wang X, Genchev GZ, et al. Multi-omics facilitated variable selection in Cox-regression model for cancer prognosis prediction[J]. Methods. 2017;124:100-7.

20. Li C, Pak D, Todem D. Adaptive lasso for the Cox regression with interval censored and possibly left truncated data[J]. Stat Methods Med Res. 2020;29(4):1243-55. 
21. Subramanian A, Tamayo P, Mootha VK, et al. Gene set enrichment analysis: a knowledge-based approach for interpreting genome-wide expression profiles[J]. Proc Natl Acad Sci U S A. 2005;102(43):15545-50.

22. Yoshihara K, Shahmoradgoli M, Martinez E, et al. Inferring tumour purity and stromal and immune cell admixture from expression data[J]. Nat Commun. 2013;4:2612.

23. Bray F, Ferlay J, Soerjomataram I, et al. Global cancer statistics 2018: GLOBOCAN estimates of incidence and mortality worldwide for 36 cancers in 185 countries[J]. CA Cancer J Clin. 2018;68(6):394-424.

24. Fujiwara N, Friedman SL, Goossens N, et al. Risk factors and prevention of hepatocellular carcinoma in the era of precision medicine[J]. J Hepatol. 2018;68(3):526-49.

25. Greten TF, Lai CW, Li G, et al. Targeted and Immune-Based Therapies for Hepatocellular Carcinoma[J]. Gastroenterology. 2019;156(2):510-24.

26. Zongyi Y, Xiaowu L. Immunotherapy for hepatocellular carcinoma[J]. Cancer Lett. 2020;470:8-17.

27. Chan SL, Mo F, Johnson PJ, et al. Performance of serum alpha-fetoprotein levels in the diagnosis of hepatocellular carcinoma in patients with a hepatic mass[J]. HPB (Oxford). 2014;16(4):366-72.

28. Dong $\mathrm{M}$, Chen $\mathrm{ZH}$, Li X, et al. Serum Golgi protein 73 is a prognostic rather than diagnostic marker in hepatocellular carcinoma[J]. Oncol Lett. 2017;14(5):6277-84.

29. Choi J, Kim GA, Han S, et al. Longitudinal Assessment of Three Serum Biomarkers to Detect Very Early-Stage Hepatocellular Carcinoma[J]. Hepatology. 2019;69(5):1983-94.

30. Ye X, Li C, Zu X, et al. A Large-Scale Multicenter Study Validates Aldo-Keto Reductase Family 1 Member B10 as a Prevalent Serum Marker for Detection of Hepatocellular Carcinoma[J]. Hepatology. 2019;69(6):2489-501.

31. Deng T, Hu B, Jin C, et al. A novel ferroptosis phenotype-related clinical-molecular prognostic signature for hepatocellular carcinoma[J]. J Cell Mol Med. 2021;25(14):6618-33.

32. Bai $Y$, Tong $W$, Xie F, et al. DNA methylation biomarkers for diagnosis of primary liver cancer and distinguishing hepatocellular carcinoma from intrahepatic cholangiocarcinoma[J]. Aging. 2021;13(13):17592-606.

33. Du Y, Ma Y, Zhu Q, et al. An m6A-Related Prognostic Biomarker Associated With the Hepatocellular Carcinoma Immune Microenvironment[J]. Front Pharmacol. 2021;12:707930.

34. Ma W, Yao Y, Xu G, et al. Identification of a seven-long non-coding RNA signature associated with Jab1/CSN5 in predicting hepatocellular carcinoma[J]. Cell Death Discov. 2021;7(1):178.

35. Liu P, Wei J, Mao F, et al. Establishment of a Prognostic Model for Hepatocellular Carcinoma Based on Endoplasmic Reticulum Stress-Related Gene Analysis[J]. Front Oncol. 2021;11:641487.ã怄.

36. Jiang $\mathrm{L}$, Zhao $\mathrm{L}, \mathrm{Bi} \mathrm{J}$, et al. Glycolysis gene expression profilings screen for prognostic risk signature of hepatocellular carcinoma[J]. Aging. 2019;11(23):10861-82.

37. D'Angiolella V, Donato V, Forrester FM, et al. Cyclin F-mediated degradation of ribonucleotide reductase M2 controls genome integrity and DNA repair[J]. Cell. 2012;149(5):1023-34. 
38. Fu J, Qiu H, Cai M, et al. Low cyclin F expression in hepatocellular carcinoma associates with poor differentiation and unfavorable prognosis[J]. Cancer Sci. 2013;104(4):508-15.

39. Serpas $L$, Chan $R$, Jiang $P$, et al. Dnase $1 / 3$ deletion causes aberrations in length and end-motif frequencies in plasma DNA[J]. Proc Natl Acad Sci U S A. 2019;116(2):641-9.

40. Guo D, Ma D, Liu P, et al. DNASE1L3 arrests tumor angiogenesis by impairing the senescenceassociated secretory phenotype in response to stress[J]. Aging. 2021;13(7):9874-99.

41. Wang S, Ma H, Li X, et al. DNASE1L3 as an indicator of favorable survival in hepatocellular carcinoma patients following resection[J]. Aging. 2020;12(2):1171-85.

42. Feldbrugge L, Jiang ZG, Csizmadia E, et al. Distinct roles of ecto-nucleoside triphosphate diphosphohydrolase-2 (NTPDase2) in liver regeneration and fibrosis[J]. Purinergic Signal. 2018;14(1):37-46.

43. Yu J, Lavoie EG, Sheung N, et al. IL-6 downregulates transcription of NTPDase2 via specific promoter elements[J]. Am J Physiol Gastrointest Liver Physiol. 2008;294(3):G748-56.

44. Craft CS, Broekelmann TJ, Mecham RP. Microfibril-associated glycoproteins MAGP-1 and MAGP-2 in disease[J]. Matrix Biol. 2018;71-72:100-11.

45. Zhu X, Cheng Y, Wu F, et al. MFAP2 Promotes the Proliferation of Cancer Cells and Is Associated With a Poor Prognosis in Hepatocellular Carcinoma[J]. Technol Cancer Res Treat. 2020;19:1079245172.

46. Wang $L$, Huang J, Jiang M, et al. Tissue-specific transplantation antigen P35B (TSTA3) immune response-mediated metabolism coupling cell cycle to postreplication repair network in no-tumor hepatitis/cirrhotic tissues (HBV or HCV infection) by biocomputation[J]. Immunol Res. 2012;52(3):258-68.

47. Zhang L, Huang Y, Ling J, et al. Overexpression of SLC7A11: a novel oncogene and an indicator of unfavorable prognosis for liver carcinoma[J]. Future Oncol. 2018;14(10):927-36.

48. Gao S, Gang J, Yu M, et al. Computational analysis for identification of early diagnostic biomarkers and prognostic biomarkers of liver cancer based on GEO and TCGA databases and studies on pathways and biological functions affecting the survival time of liver cancer[J]. BMC Cancer. 2021;21(1):791.

49. Zhang R, Gao X, Zuo J, et al. STMN1 upregulation mediates hepatocellular carcinoma and hepatic stellate cell crosstalk to aggravate cancer by triggering the MET pathway[J]. Cancer Sci. 2020;111(2):406-17.

50. Matsusaka K, Fujiwara Y, Pan C, et al. alpha1-acid glycoprotein enhances the immunosuppressive and protumor functions of tumor-associated macrophages[J]. Cancer Res, 2021.

51. Wang Y, Liu T, Tang W, et al. Hepatocellular Carcinoma Cells Induce Regulatory T Cells and Lead to Poor Prognosis via Production of Transforming Growth Factor-beta1[J]. Cell Physiol Biochem. 2016;38(1):306-18.

52. Pena-Asensio J, Calvo H, Torralba M, et al. Anti-PD-1/PD-L1 Based Combination Immunotherapy to Boost Antigen-Specific CD8(+) T Cell Response in Hepatocellular Carcinoma[J]. Cancers (Basel), 2021,13(8). 
53. Sangro B, Gomez-Martin C, de la Mata M, et al. A clinical trial of CTLA-4 blockade with tremelimumab in patients with hepatocellular carcinoma and chronic hepatitis C[J]. J Hepatol. 2013;59(1):81-8.

54. Zhu AX, Finn RS, Edeline J, et al. Pembrolizumab in patients with advanced hepatocellular carcinoma previously treated with sorafenib (KEYNOTE-224): a non-randomised, open-label phase 2 trial[J]. Lancet Oncol. 2018;19(7):940-52.

55. Sun L, Gao F, Gao Z, et al. Shed antigen-induced blocking effect on CAR-T cells targeting Glypican-3 in Hepatocellular Carcinoma[J]. J Immunother Cancer, 2021,9(4).

56. Yang XD, Kong FE, Qi L, et al. PARP inhibitor Olaparib overcomes Sorafenib resistance through reshaping the pluripotent transcriptome in hepatocellular carcinoma[J]. Mol Cancer. 2021;20(1):20.

57. Dai X, Guo Y, Hu Y, et al. Immunotherapy for targeting cancer stem cells in hepatocellular carcinoma[J]. Theranostics. 2021;11(7):3489-501.

58. Liu J, Yi J, Zhang Z, et al. Deoxyribonuclease 1-like 3 may be a potential prognostic biomarker associated with immune infiltration in colon cancer[J]. Aging. 2021;13(12):16513-26.

59. Wang $L$, Huang J, Jiang $M$, et al. Tissue-specific transplantation antigen P35B (TSTA3) immune response-mediated metabolism coupling cell cycle to postreplication repair network in no-tumor hepatitis/cirrhotic tissues (HBV or HCV infection) by biocomputation[J]. Immunol Res. 2012;52(3):258-68.

60. Tu K, Li J, Mo H, et al. Identification and validation of redox-immune based prognostic signature for hepatocellular carcinoma[J]. Int J Med Sci. 2021;18(9):2030-41.

61. He M, Li Q, Zou R, et al. Sorafenib Plus Hepatic Arterial Infusion of Oxaliplatin, Fluorouracil, and Leucovorin vs Sorafenib Alone for Hepatocellular Carcinoma With Portal Vein Invasion: A Randomized Clinical Trial[J]. JAMA Oncol. 2019;5(7):953-60.

62. Zhang $X, H u B$, Sun YF, et al. Arsenic trioxide induces differentiation of cancer stem cells in hepatocellular carcinoma through inhibition of LIF/JAK1/STAT3 and NF-kB signaling pathways synergistically[J]. Clin Transl Med. 2021;11(2):e335.

\section{Figures}


Figure 1
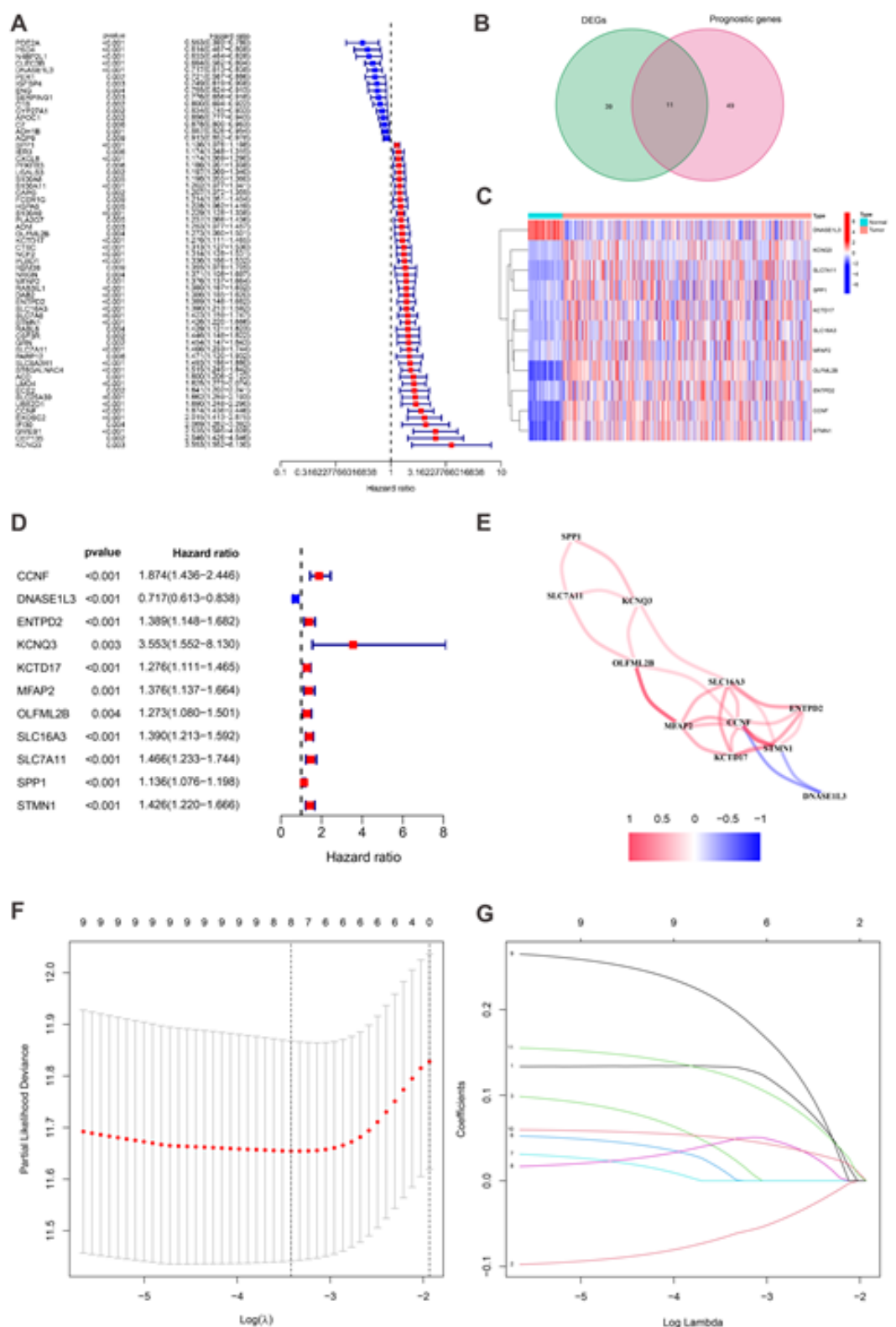

\section{Figure 1}

Screening of immune and inflammatory response related genes in the TCGA cohort. (A) Distribution of LASSO coefficients of inflammation-related genes in breast cancer. (B) Partial likelihood deviance against $\log (\lambda)$ is plotted. The first vertical dashed line representatives the I value with minimum error.(C-D)KaplanMeier curves shows the Survival difference between high-risk group and low-risk group in the TCGA and ICGCcohort. 
Figure 2
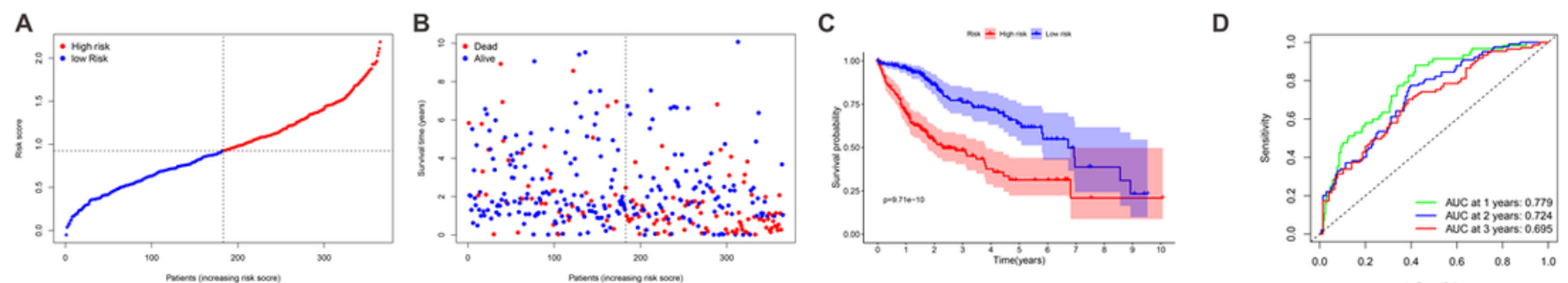

$E$
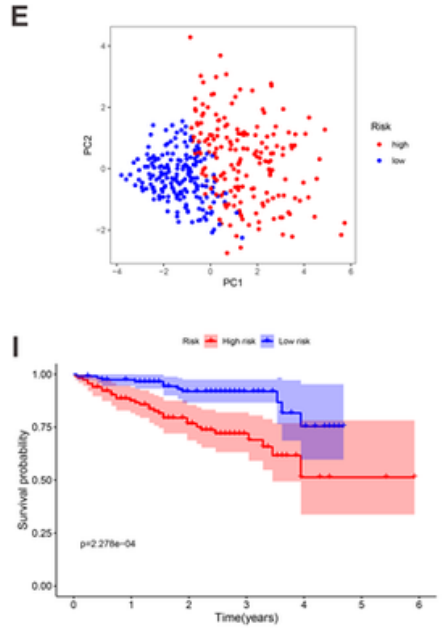

$\mathbf{F}$

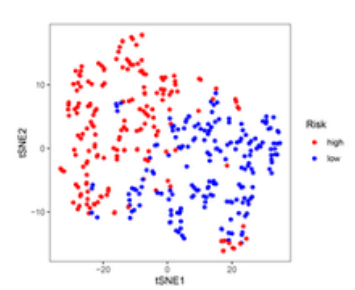

$\mathrm{J}$

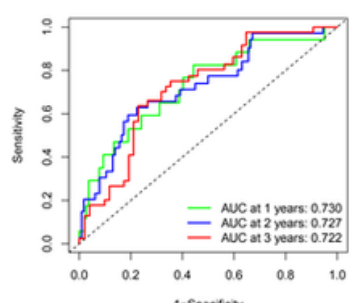

G.

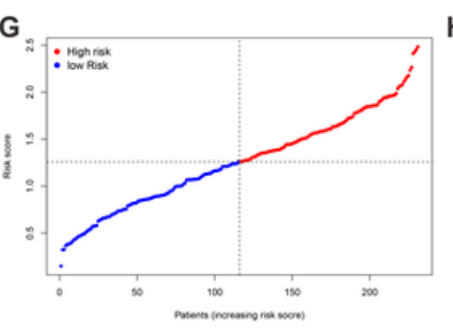

K

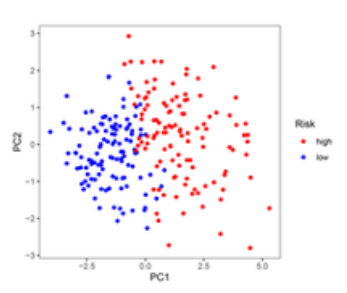

H

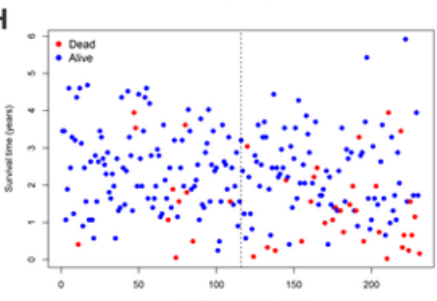

L

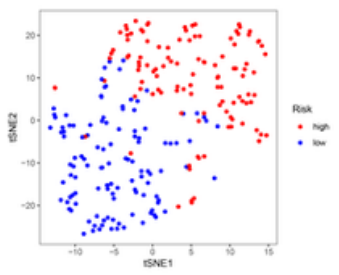

Figure 2

Prognosis of 8 genetic characteristic models in TCGA and ICGC cohort. TCGA queues (A, B, C, D, E, F), ICGC queues $(G, H, I, J, K, L)$. (A, G) The distribution and median of the risk score. $(B, H)$ The status of OS and the distribution of risk scores. $(C, I)$ Kaplan-Meier analysis survival rates for patients in high-risk and low-risk groups. $(D, J)$ AUC time-dependent ROC curve evaluates the prognosis model for OS. (E,K) PCA diagram. $(F, I) t-S N E$ analysis.
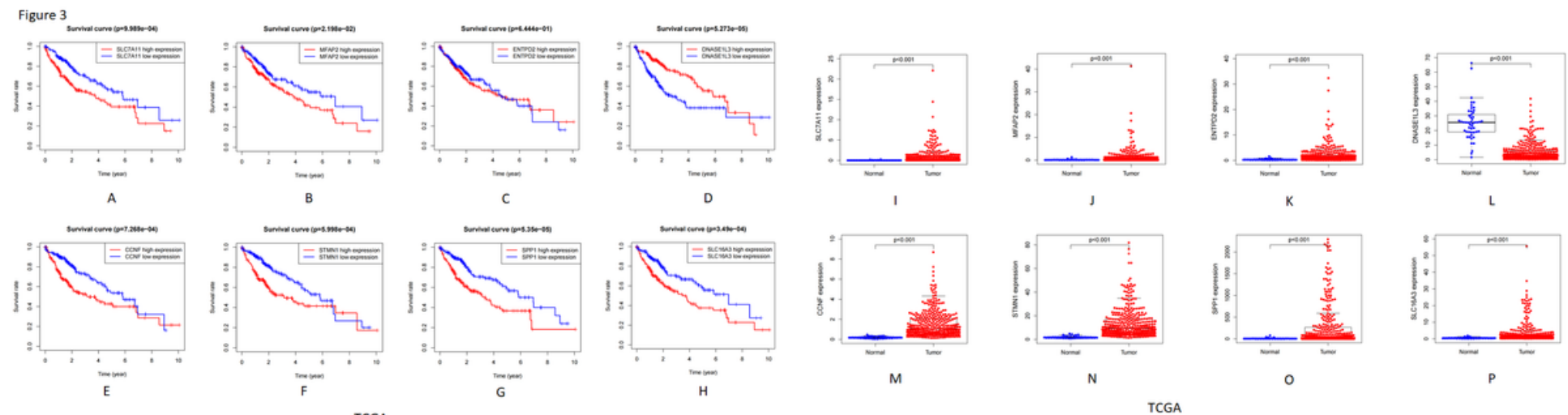

Figure 3

8 genes express and OS of HCC in TCGA cohort. The level of expression of 8 genes in tumor tissue (A-H) and the Kaplan-Meier curve of OS in patients with high- and low-risk groups (I-P)in TCGA cohort. 
Figure 4

TCGA

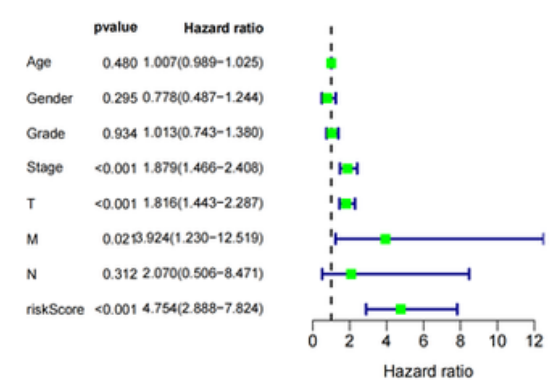

A

ICGC

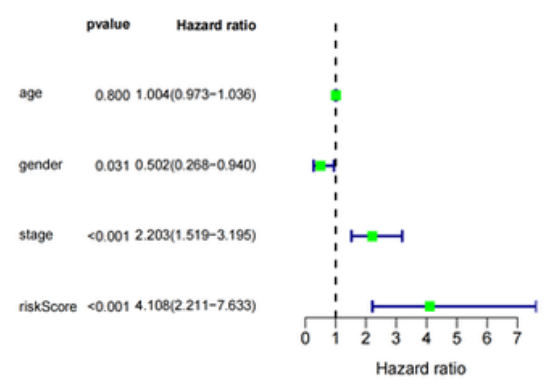

D

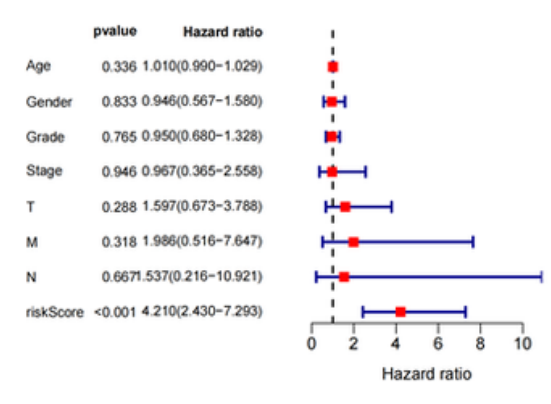

B

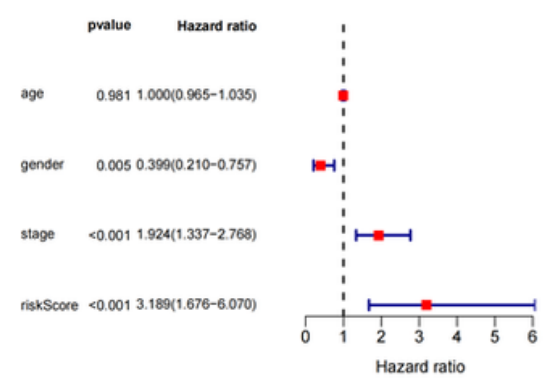

E

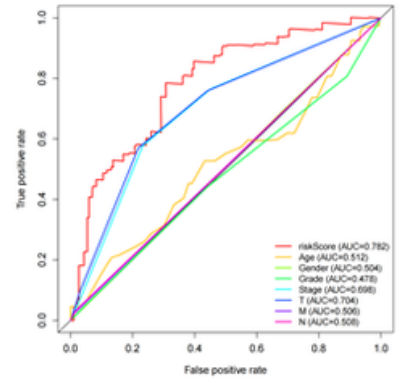

C

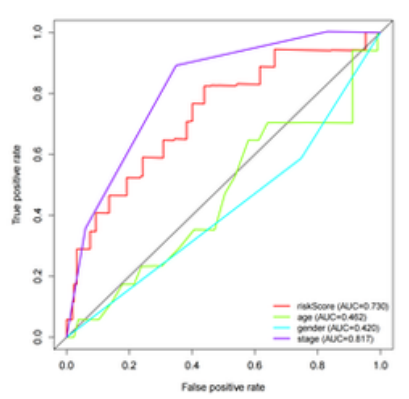

F

\section{Figure 4}

Screening OS-related single factors to compare the prognosis accuracy of risk scores and clinical pathology factors. TCGA cohort $(A, B, C)$, ICGC cohort (D, E, F). (A,D) Screening OS-related factors using Univariate Cox regression analysis. $(B, E)$ Filter os-related factors using Multivariate Cox regression analysis. (C,F) The prognosis accuracy of risk scoring combined with clinical pathological characteristics was compared using time-dependent ROC curves. 
Figure 5

TCGA

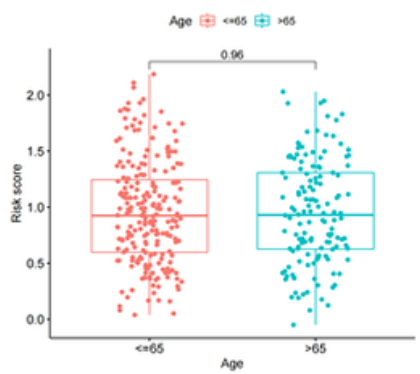

A

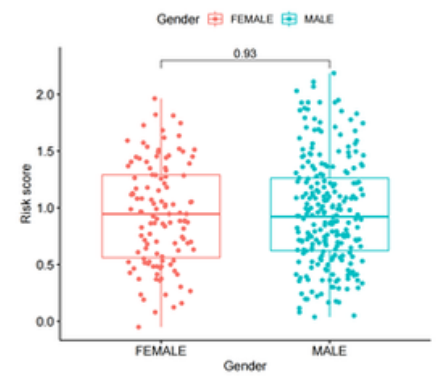

B

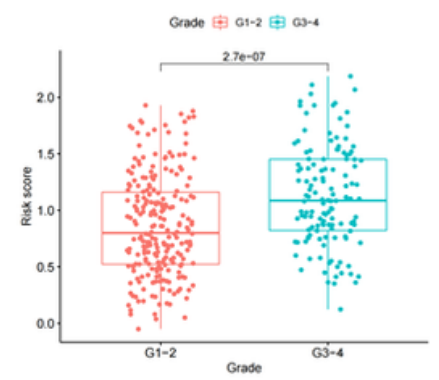

C

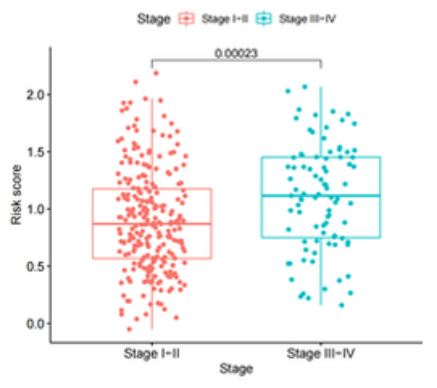

D

ICGC

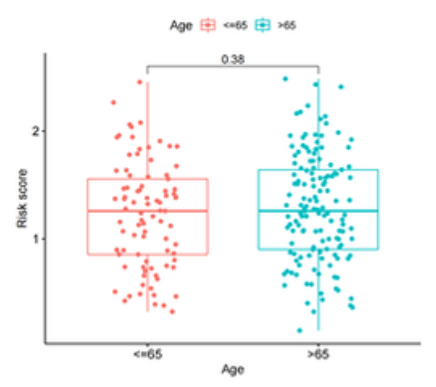

E

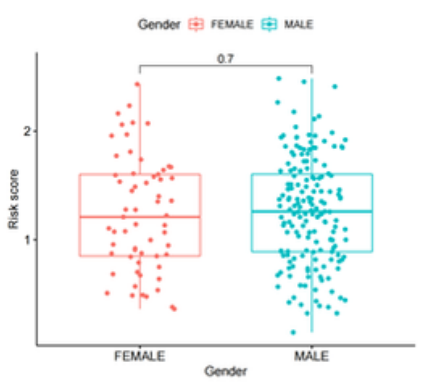

F

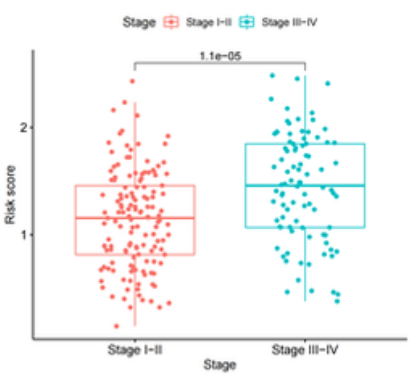

G

\section{Figure 5}

Risk scores for high and low risk groupings divided with clinical characteristics. TCGA cohort (A-D), ICGC cohort (E-F). (A, E) Age. (B, F) Gender. (C) Grade. (D, G) Stage.

Figure 6

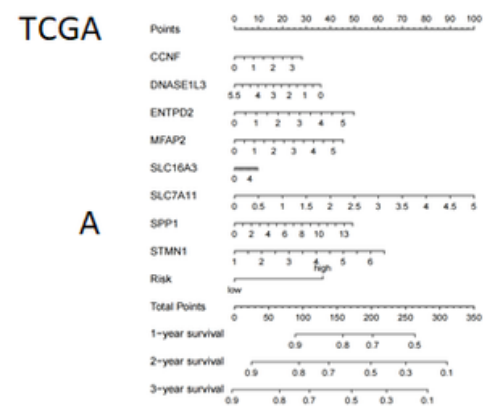

ICGC

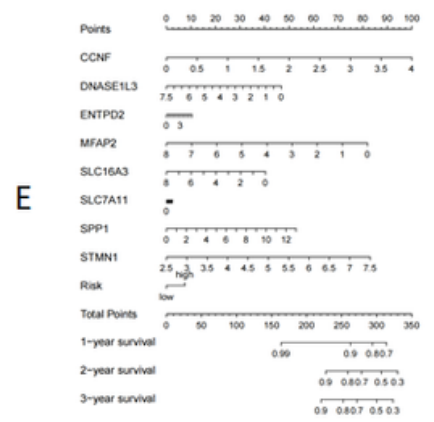

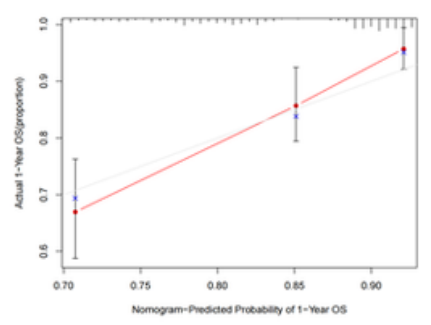

B

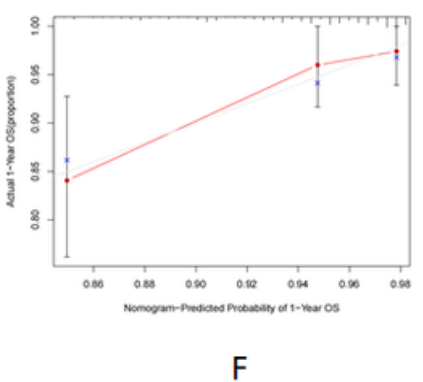

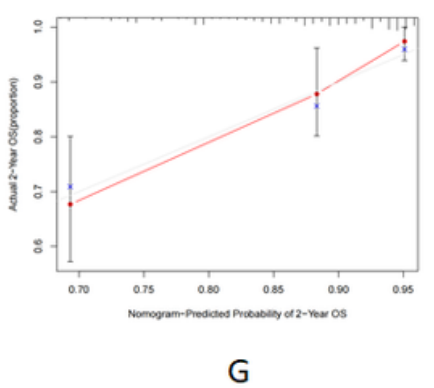
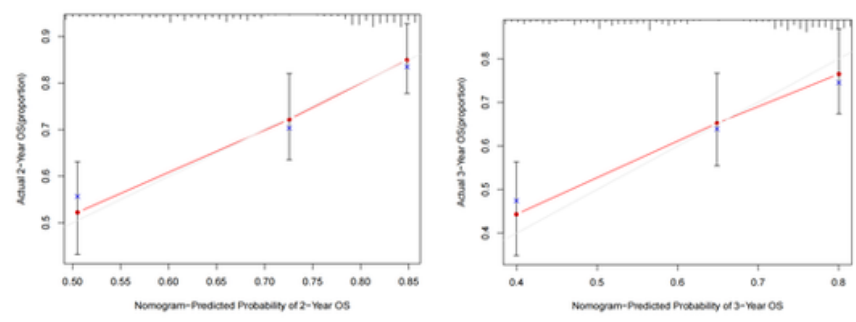

C

D

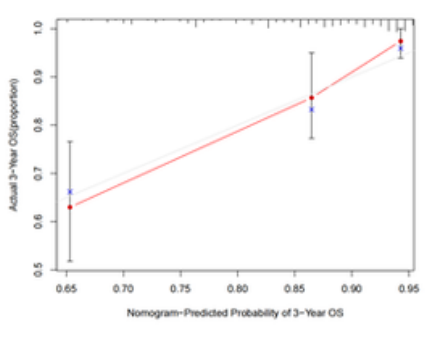

$\mathrm{H}$ 


\section{Figure 6}

Nomogram based on the prognosis characteristics of the 8 genes is in the TCGA and ICGC cohort. (A-D) and ICGC (D-H) cohort. (A, E): Build a nomogram model of 8 genes and high and low risk to predict one, two, and three years of survival. The calibration chart shows that the predicted survival rate is consistent with the actual survival rates for 1 year $(B, F), 2$ years $(C, G)$, and 3 years $(D, H)$.

Figure 7
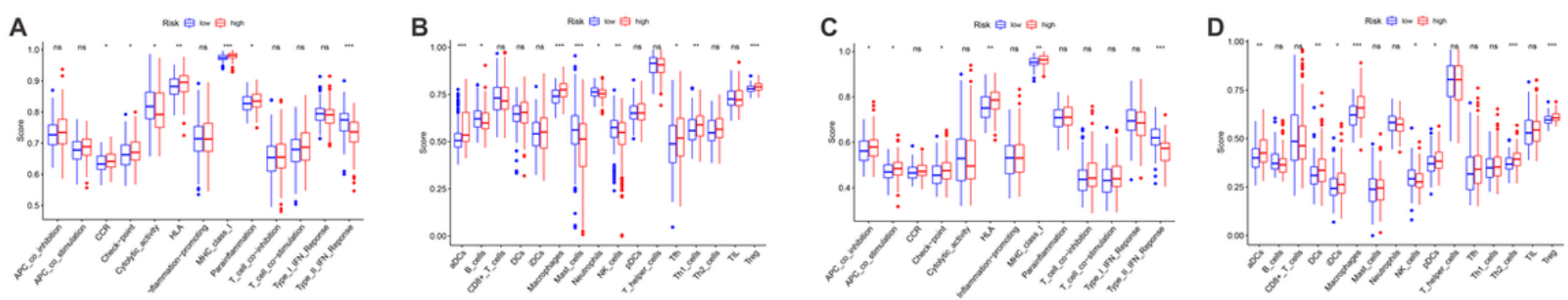

$E$

Immune subtype

G

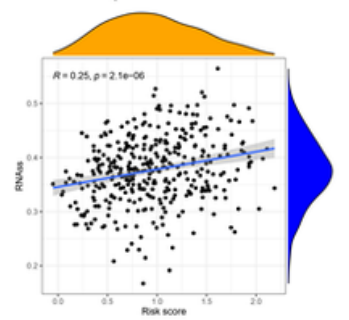

H

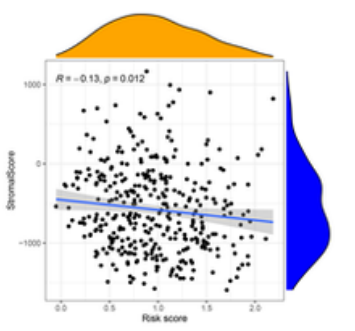

$\mathrm{F}$

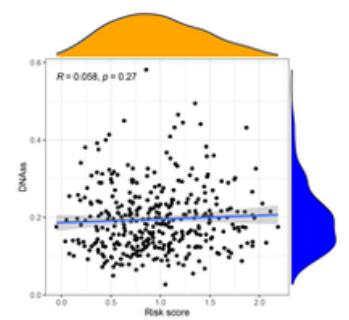

I

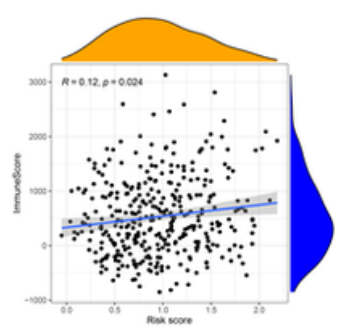

\section{Figure 7}

Relationship between immune status and risk score and tumor microenvironment in the high-and low-risk groups. TCGA cohort (A, B), ICGC cohort (C, D).13 immuno-related functions scored (A, C) and 16 immune cells $(B, D)$. (E) Comparison of risk scores for different immuno-immersion subtypes. (F-I) Relationship between risk scores and DNAss, RNAss, Stromal scores, and immune scores. P values were showed as: ns, not significant; ${ }^{\mathrm{P}}<0.05 ; * \star \mathrm{P}<0.01 ; * \star \star \mathrm{P}<0.001$. 


\section{Figure 8}

\section{Cancer: LIHC}

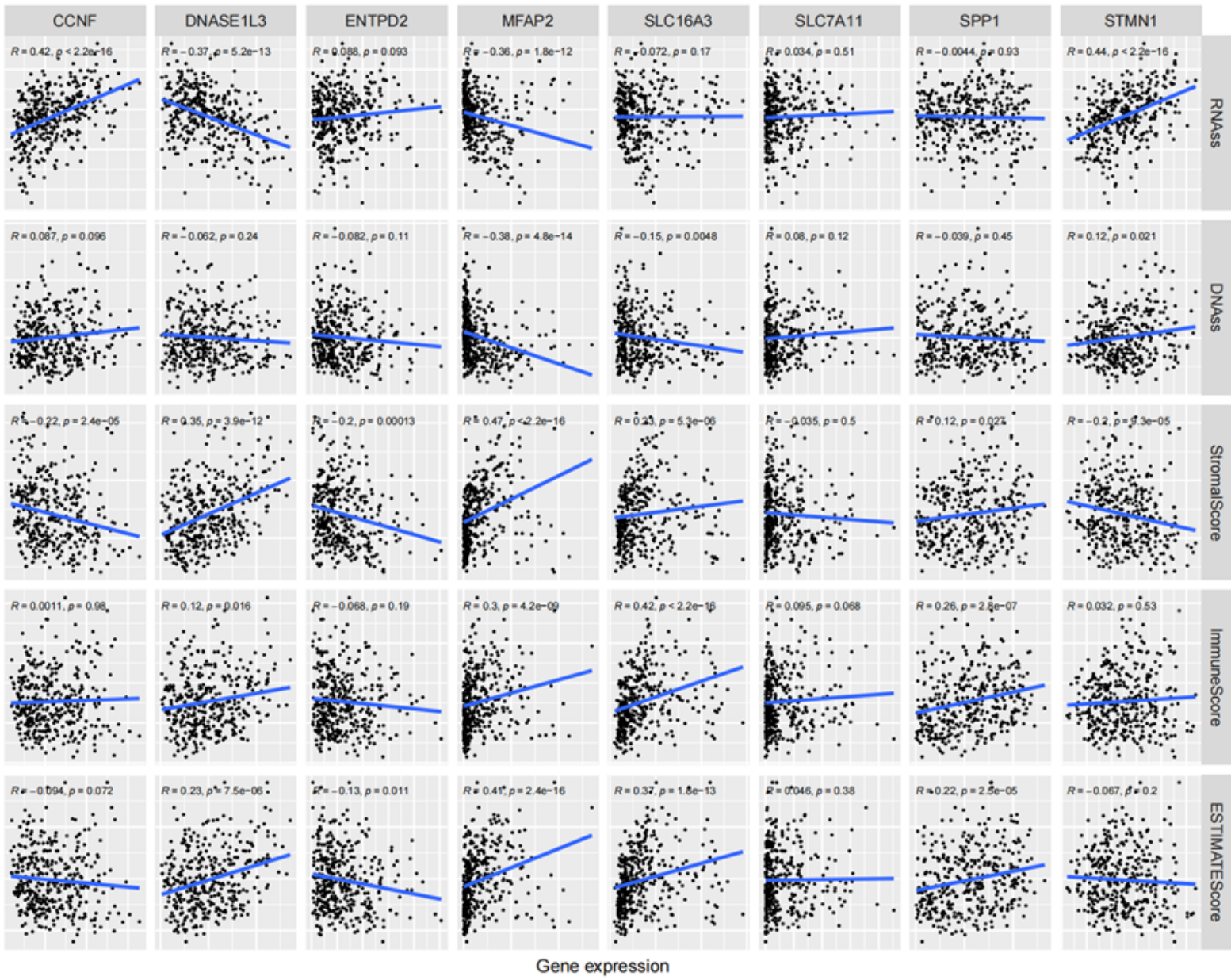

\section{Figure 8}

Prognostic gene expression correlation with stromal score and immune score. CCNF, DNASE1L3, ENTPD2, MFAP2, SLC16A3, SPP1, STMN1 have a strong correlation with Stromal score. DNASE1L3, MFAP2, SLC16A3, and SPP1 are closely related to the immune score, 
Figure 9

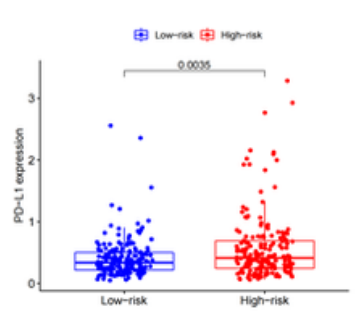

A

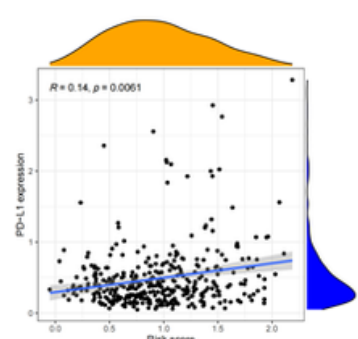

F

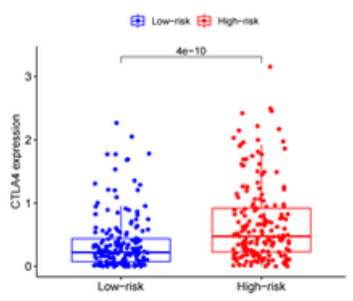

B

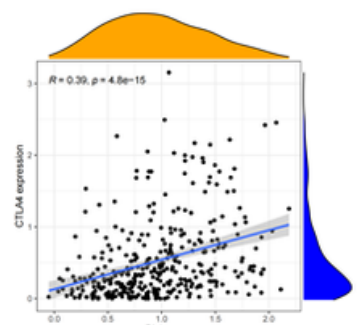

G

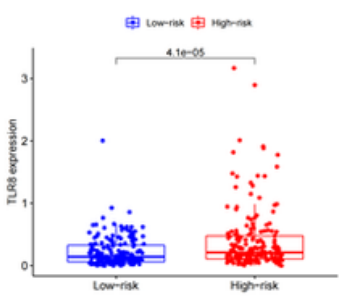

C

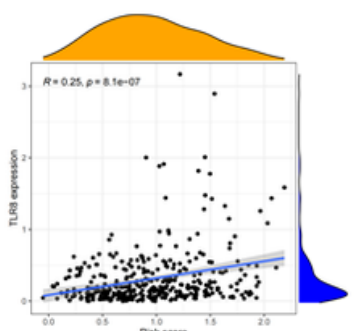

H

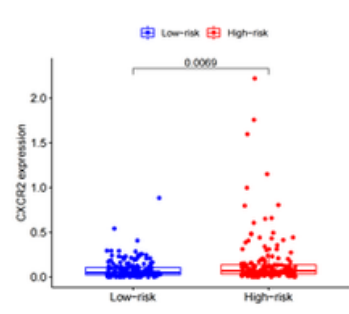

D

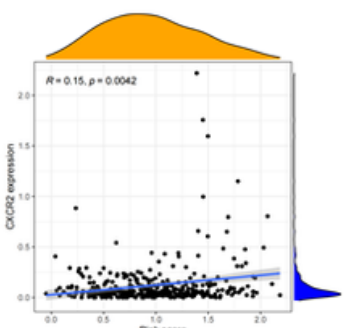

I

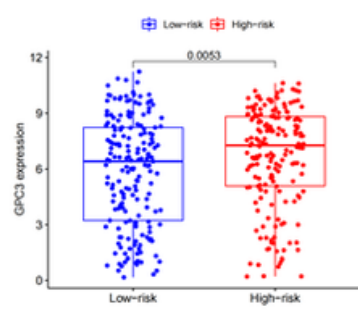

E

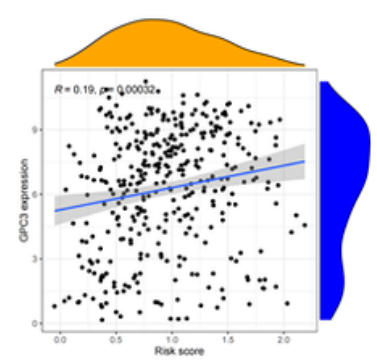

J

\section{Figure 9}

The expression levels of PD-L1, CTLA4, TLR8, CXCR2 and GPC3 in the high and low risk groups. as well as correlation analysis of risk scores and PD-L1, CTLA4, TLR8, CXCR2 and GPC3 expressions. $\triangle A$ AF区PD-

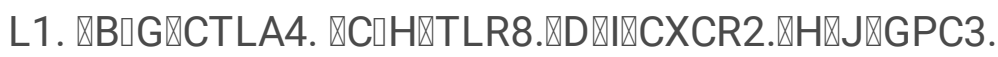

Figure 10

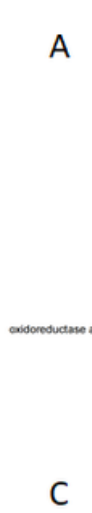

C
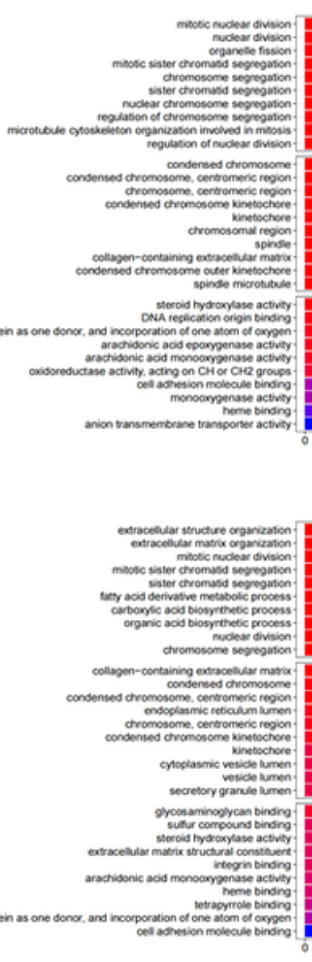
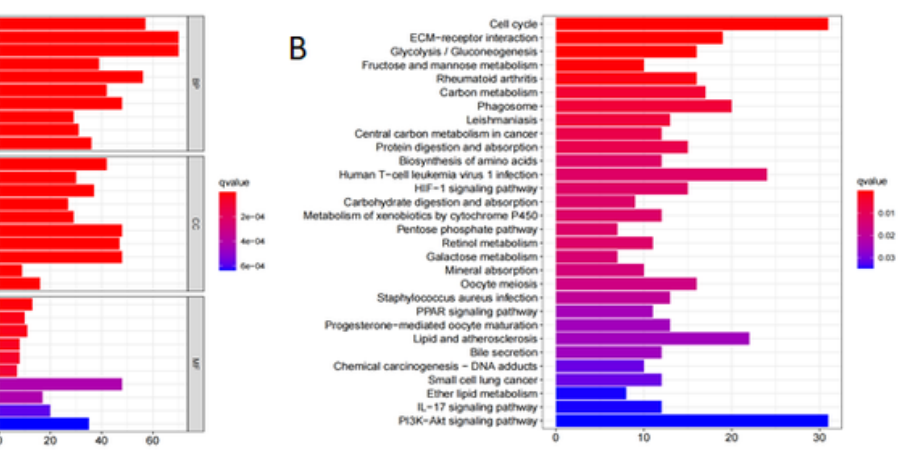

D
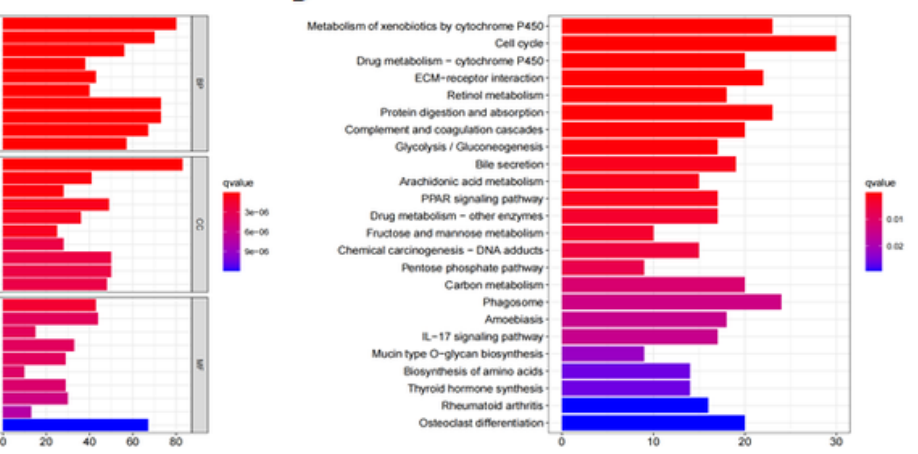
Figure 10

Functional enrichment analysis is based on DEGs between high and low risk groups in the TCGA and ICGC cohort. (A囚C) GO, Gene Ontology. (B,D) KEGG, Kyoto Encyclopedia of Genes and Genomes.

Figure 11

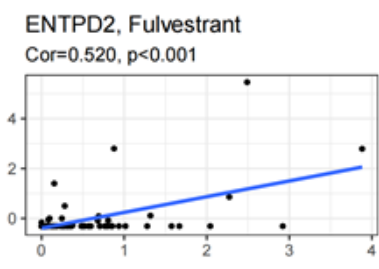

DNASE1L3, Isotretinoin Cor $=0.449, p<0.001$

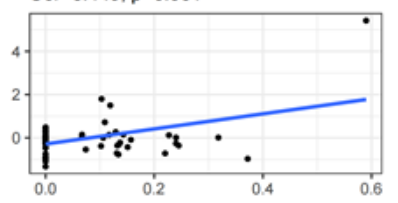

DNASE1L3, Megestrol acetate Cor $=0.417, p<0.001$
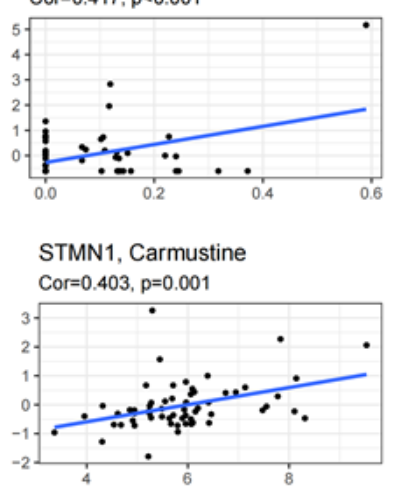
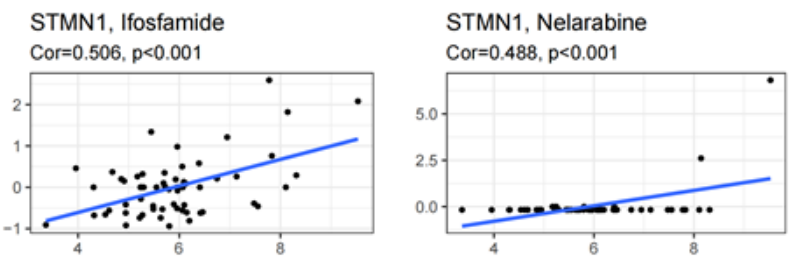

DNASE1L3, Oxaliplatin

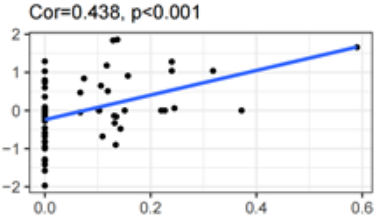

STMN1, Hydroxyurea

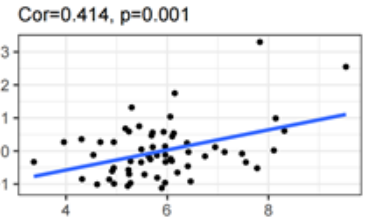

SLC7A11, Ixazomib citrate Cor $=-0.401, p=0.002$

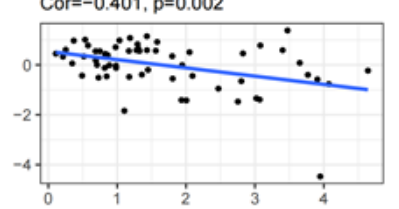

SLC7A11, Arsenic trioxide

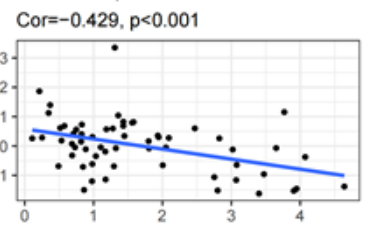

STMN1, Dexamethasone DecadroI Cor $=0.408, p=0.001$

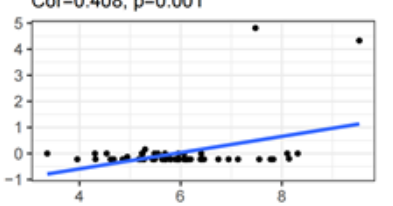

DNASE1L3, Fluphe0zine Cor $=0.400, p=0.002$

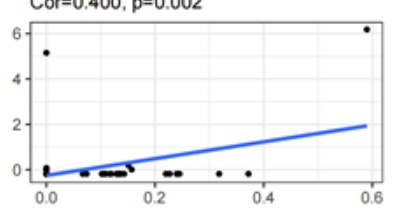

MFAP2, Bisacodyl, active ingredient Cor $=0.462, p<0.001$

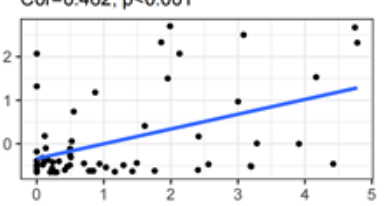

DNASE1L3, Imiquimod Cor $=0.426, p<0.001$

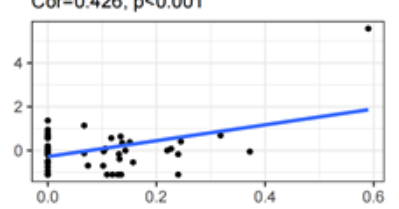

DNASE1L3, Epirubicin

Cor $=0.405, p=0.001$

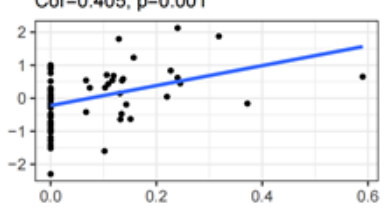

STMN1, Parthenolide Cor $=0.391, p=0.002$

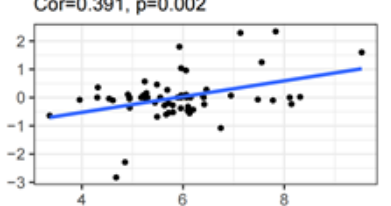

\section{Figure 11}

Scatter plot of the relationship between 8 related prognostic gene expression and drug sensitivity. 